\title{
« Le Muscle et le Vouloir» Les sports dans les collèges classiques masculins au Québec, 1870-1940 ${ }^{1}$
}

\section{Christine Hudon}

Dans les années 1870, les sports modernes font leur apparition dans les collèges classiques pour garçons. Ils ont pour fonction de contribuer à la maîtrise des pulsions et à la formation du caractère. L'introduction de ces activités sportives découle essentiellement de deux facteurs : la mutation du rapport au corps et la transformation de l’idéal viril qui l'accompagne, ainsi que le renouvellement de la spiritualité catholique. L'article met en relief la perméabilité des collèges classiques aux influences externes. Il montre que le sport devient un instrument d'une pédagogie religieuse faisant la promotion d'une spiritualité aux accents virils, non pas fondée sur le mépris du corps, mais sur une ascèse visant à le fortifier.

Modern sports appeared in classical colleges for boys in the 1870s. Their function was to contribute to character formation and to the control of physical drives. The introduction of these sports activities was based on two factors: the changing relationship to the body and the transformation of the accompanying masculine ideal, and the renewal of Catholic spirituality. This article highlights the susceptibility of classical colleges to external influences. It demonstrates that sports became an instrument in a religious education that promoted a spirituality with masculine undertones, based not on a contempt for the body but on an asceticism that sought to strengthen it.

À la fin des années 1930, les élèves du séminaire Saint-CharlesBorromée, à Sherbrooke, entonnent le Chant des gymnases, glorifiant le corps musclé auquel sont associées des vertus physiques et morales :

Ici va prévaloir

La souple stratégie

Qui bombe d'énergie

Le Muscle et le Vouloir

1 Cet article a été réalisé grâce au soutien financier du FQRSC et du CRSH. Il a bénéficié des suggestions de Louise Bienvenue et du travail de recherche en archives qu’ont effectué Tania Perron, Émilie Létourneau, François Morin, Guillaume BreaultDuncan, Alexandre Janelle-Blanchette, Pascal Allard et Mathieu Béchard.

(CHistorical Studies in Education / Revue d'histoire de l'éducation 17, 2 (2005): 243-63 
Marchons droit (bis) / C'est la loi (bis)

Et vive l'hygiène

Qui donne à l'âme saine

Un corps moins maladroit

Saluons (bis) la santé / La santé qui se double d'agilité (bis)².

Cet hymne alliant une âme et un corps sains n'est pas sans rappeler une marche militaire. Il est caractéristique de l'émergence et de l'essor, au $19^{\mathrm{e}}$ siècle et au début du siècle suivant, d'un nouvel idéal masculin perceptible jusque dans le discours et les pratiques éducatives des collèges classiques québécois. Le présent article s'intéresse au contexte d'élaboration de ce rapport renouvelé au corps, et à l'avènement des sports modernes auquel il a contribué. À la fin du $18^{\mathrm{e}}$ et au début du $19^{\text {e }}$ siècle, en effet, les jeux traditionnels sont graduellement remplacés par des disciplines codifiées aux formes diverses (athlétisme, tennis, golf, courses cyclistes, football, etc.). À l'instar de plusieurs auteurs, nous nous intéresserons à la montée de ces sports modernes, compris ici comme l'ensemble des jeux compétitifs obéissant à des règles strictes et mettant en œuvre des forces du corps en vue de la réalisation d'une performance ${ }^{3}$. Nous observerons leur introduction dans les collèges en portant attention aux facteurs qui ont favorisé leur implantation.

\section{Un esprit « chrétien » opposé aux sports ?}

Deux questions dominent l'historiographie canadienne et québécoise consacrée à l'émergence du sport à l'école. Ainsi, certaines publications ont analysé les rapports entre le développement du sport et la construction de l'identité nationale ${ }^{4}$. Elles ont montré que les sports, tout en contribuant à la formation du sentiment national, ont connu une diffusion inégale selon les régions et les provinces. S’agissant de l'éventail des exercices et des disciplines en vogue, Canadiens français et Canadiens anglais auraient puisé à des influences culturelles en partie différentes, certains jeux d'origine britannique, par exemple le

2 Archives du séminaire de Sherbrooke (désormais ASS), Chroniques du Séminaire 1929-1941, 207.

3 Sur la multiplicité des acceptions données au terme, lire entre autres Jacques Defrance, Sociologie du sport (Paris : La Découverte, 1997); Jean-Marie Brohm, Sociologie politique du sport (Paris : Jean-Pierre Delarge, 1976); et aussi l'ouvrage pionnier et d'une influence considérable de Georges Hébert, Le Sport contre l'Éducation physique (Paris : Vuilbert, 1946 (1925)), 6-7.

4 Donald Guay, La conquête du sport. Le sport et la société québécoise au XIXe siècle (Outremont : Lanctôt, 1997); Gilles Janson, Emparons-nous du sport : les Canadiens français et le sport au XIX ${ }^{e}$ siècle (Montréal : Guérin, 1995); Alan Metcalfe, Canada Learns to Play. The Emergence of Canadian Sport, 1807-1914 (Toronto : McClelland and Stewart, 1987). 
rugby, le cricket et le soccer, ayant fait une percée très limitée dans les milieux francophones. Quelques recherches se sont par ailleurs intéressées au rôle et à l'attitude des acteurs sociaux et des institutions, principalement l'Église et l'État, dans le développement des pratiques et des associations sportives ${ }^{5}$.

Ces études, comme, du reste, celles consacrées à la question de l'identité nationale, ont fait état de la méfiance du clergé catholique à l'égard du sport à la fin du $19^{\mathrm{e}}$ siècle et au début du $20^{\mathrm{e}}$ siècle. JeanPaul Massicotte et Claude Lessard notent par exemple que l'Église condamne le sport jusque dans les années $1930^{6}$, tandis que dans son livre La Conquête du sport, consacré au $19^{\mathrm{e}}$ siècle et aux premières décennies du $20^{\mathrm{e}}$ siècle, Donald Guay oppose ce qu'il appelle « l'esprit chrétien » à " l'esprit sportif ». D'après Guay, l'un et l'autre esprits poursuivent des objectifs contradictoires : " l'esprit chrétien » est fondé sur le culte de l'âme, la force intérieure, le rapport à Dieu, l'humilité, la coopération, le partage et le renoncement, alors que " l'esprit sportif » fait la promotion du culte du corps, de la force extérieure, du rapport à l'homme, de la gloire, de la compétition, de la domination et du plaisir ${ }^{7}$. Selon Donald Guay, la stratégie pédagogique des clercs visant « à développer la coopération, le partage, l'entraide, le sens communautaire, bref l'altruisme " a longtemps fait mauvais ménage avec les pratiques sportives fondées sur la compétition, la combativité, le désir de vaincre et la valorisation des meilleurs ${ }^{8}$.

Cette interprétation, qui réfère implicitement à une conception augustinienne du corps, repose à notre avis sur une double méprise. D'abord, elle suggère qu'il y a un idéal chrétien intemporel et un discours unique, au sein de l'Église, sur le rapport au corps et la pratique des exercices corporels et des sports de compétition. Pourtant, les moralistes catholiques ont offert une diversité d'opinions sur la question. Des recherches ont également montré l'ouverture graduelle du clergé aux activités physiques et sportives. Tout en signalant les réticences et les débats que les exercices corporels et les amusements sportifs ont suscités parmi les clercs, Michel Lagrée a par exemple mis en lumière le rôle du clergé breton dans la diffusion de plusieurs activités et la mise sur pied d'organisations sportives à la fin du $19^{\mathrm{e}}$

5 En plus des ouvrages mentionnés cités ci-dessus, Jean-Paul Massicotte et Claude Lessard, "L'Église et le loisir au Québec au XX $\mathrm{X}^{\mathrm{e}}$ siècle ", Canadian Journal of the History of Sport/Revue canadienne de l'histoire du sport, 13, 2 (décembre 1982) : 45-55; Michel Bellefleur, L'Église et le loisir au Québec avant la Révolution tranquille (Sillery: Presses de l’Université du Québec, 1986).

6 J.-P. Massicotte et C. Lessard, "L'Église et le loisir au Québec au XX ${ }^{\mathrm{e}}$ siècle ", Canadian Journal of the History of Sport/Revue canadienne de l'histoire du sport, 13, 2 (décembre 1982) : 45-55.

7 Guay, La conquête du sport, 186.

8 Ibid., 188. 
siècle et au début du $20^{\mathrm{e}}$ siècle ${ }^{9}$. Selon Lagrée, le développement des clubs sportifs d'obédience catholique a été favorisé par certains ecclésiastiques éminents, tel le cardinal Mercier, archevêque de Vannes, qui en a encouragé la propagation dans les collèges et les séminaires. Au début du $20^{\mathrm{e}}$ siècle, le pape Pie X lui-même donna sa bénédiction aux sociétés sportives italiennes. Ses successeurs Pie XI et Pie XII développèrent quant à eux un discours catholique plus ouvert encore aux exercices corporels, auxquels étaient dorénavant attribués des bénéfices d'ordre spirituel, du moins en ce qui a trait aux pratiques masculines, l'Église continuant d'entretenir une attitude suspicieuse à l'égard du sport féminin ${ }^{10}$.

Cette interprétation se méprend également sur la stratégie pédagogique des clercs. En effet, les pratiques éducatives des prêtres sont loin de rejeter la compétition. La notion de concurrence est plutôt inscrite au cœur même de la pédagogie des institutions religieuses d'enseignement qui valorisent l'émulation, les concours, les classements et les prix ${ }^{11}$. L'enseignement des collèges classiques ne s'oppose donc pas à la notoriété, à la gloire et à la domination. Certes, il cultive l'humilité devant Dieu et l'obéissance aux supérieurs, mais il vise aussi à former une élite. À cette fin, il couronne les premiers, leur octroie des congés de devoir et leur remet des livres en récompenses. À l'intérieur des classes, les maîtres font connaître publiquement les résultats des examens et des compositions, félicitent les meilleurs et se moquent parfois des plus faibles. Aux $19^{\mathrm{e}}$ et $20^{\mathrm{e}}$ siècles, les maisons d'éducation recourent aussi aux journaux pour annoncer les succès des plus méritants, dans le but, notamment, de se démarquer des institutions concurrentes. Enfin, toutes sortes de privilèges et de récompenses couronnent les élèves exemplaires. Au collège de Sherbrooke, par exemple, une croix d'argent, portant l'inscription " Témoignage d'Application et de Bonne Conduite ", est portée pendant quinze jours par celui qui se fait remarquer par sa fidélité à accomplir son devoir ${ }^{12}$.

En raison de cette méprise relative, d'une part, au discours chrétien sur le corps et, d'autre part, à la pédagogie des clercs, il

9 Michel Lagrée, Religion et cultures en Bretagne (1850-1950)(Paris : Fayard, 1992), 405-431. Aussi : B. Dubreuil, « Naissance du sport catholique », Recherches, 43 (1980): 221-251; Benoît Caritey, " Catholicisme, patriotisme et sport en Alsace (1898-1939) », Cahiers d'histoire, 88, 3 ( $3^{\mathrm{e}}$ trimestre 2002); Bruno Dumons, Gilles Pollet, Muriel Berjat, " L’Église, un instigateur actif » dans Naissance du sport moderne (Lyon : La Manufacture, 1987), 119-130.

10 Pour un aperçu de l'attitude de l'Église catholique à l'égard du sport, lire J. Gritti, "Sport » dans Catholicisme : hier, aujourd'hui, demain, tome quatorzième (Paris : Letouzey et Ané, 1996), 404-414.

11 Voir Galarneau, Les collèges classiques au Canada français (1620-1970) (Montréal : Fides, 1978), 193-195.

12 ASS, «La croix d'honneur ", Annuaire du Séminaire de Sherbrooke, N 1, Année académique 1875-1876, p.22. 
apparaît important de réexaminer l'attitude du clergé à l'égard du sport dans les collèges classiques. Notre hypothèse est la suivante. Au cours de la période étudiée, soit les années 1870 à 1940, on ne saurait qualifier d'antinomiques les rapports entre la pédagogie religieuse et le sport. Ce dernier devient plutôt l'instrument d'une pratique éducative qui se modernise et qui développe une ascèse visant non pas à mortifier le corps, mais à le fortifier. Comme l'ont fait remarquer les historiens du sport ${ }^{13}$, les programmes scolaires de cette période n'intègrent guère l'éducation physique au sens que lui a donné, dans les années 1920, le Français Georges Hébert ${ }^{14}$. Néanmoins, à la faveur d'un contexte social, politique et religieux de plus en plus attentif au corps et à la culture physique, les collèges proposent à leurs élèves, à compter de la décennie 1870, une gamme croissante d'activités sportives, auxquelles sont attribuées des fonctions bien précises. Les jeux et les exercices que pratiquent les collégiens pendant les récréations, mais surtout lors des congés, s'insèrent, en fait, dans ce que Jacques Defrance appelle une orientation utilitariste du sport, par opposition à une orientation qui pourrait être qualifiée d'hédoniste ${ }^{15}$.

Dans cette optique, les pratiques sportives dans les institutions d'enseignement secondaire pour garçons poursuivent principalement deux objectifs : elles offrent un délassement acceptable, de loin préférable à l'oisiveté, et elles contribuent à la formation globale des élèves. Du point de vue de plusieurs prêtres enseignants, le sport procure en effet une détente aux collégiens qui passent de nombreuses heures à étudier. Il permet d'occuper leur imagination, canalise leur énergie sexuelle et, surtout, forme le caractère en instillant le courage, l'endurance, la discipline, la soumission aux règles et l'acceptation joyeuse de l’échec. Par le corps, on rejoint l'âme.

\section{Les activités physiques et les jeux dans les collèges classiques}

On sait l'importance qu'a eue le collège classique dans l'histoire de l'enseignement au Québec. Cet espace de socialisation clos sur luimême, privilégiant la formule de l'internat, a joué un rôle majeur dans le processus éducatif. Il a contribué à former des générations

13 En particulier, Donald Guay, L'histoire de l'éducation physique au Québec : conceptions et événements (1830-1980) (Chicoutimi : G. Morin, 1980).

14 L'éducation physique recouvre, pour lui, « toute action méthodique, progressive et continue, de l'enfance à l'âge adulte ayant pour objet d'assurer le développement physique intégral; d'accroître les résistances organiques; de mettre en valeur les aptitudes dans tous les genres d'exercices naturelles et utilitaires indispensables (...); de développer l'énergie et toutes les autres qualités d'action ou viriles, enfin de subordonner tout l'acquis, physique et viril, à une idée morale dominante : l'altruisme ». Hébert, Le Sport..., 13.

15 Jacques Defrance, «Politisations et apolitismes sportifs, en France et au Québec, 1930-1960 », Bulletin d'histoire politique, 11, 2 (hiver 2003) : 15-29. 
d'hommes qui ont constitué, dans les sphères les plus diverses - de l'agriculture à la prêtrise, en passant par le commerce, l'industrie, le droit, la médecine et la science - la catégorie des détenteurs de savoir et de pouvoir. Conçu pour offrir une formation humaniste générale, le collège accueillait des élèves généralement âgés de 11 à 18 ans pour des scolarités à durée variable. Certains d'entre eux suivaient le cursus complet, des Éléments à la Philosophie, passage obligé pour entrer au Grand Séminaire et voie d'accès privilégiée à des études universitaires. D'autres ne réalisaient que les trois ou quatre premières années d'étude, et parfois beaucoup moins. Ils y acquéraient un bagage de connaissances académiques et d'habitudes culturelles - un capital symbolique en quelque sorte - susceptibles de nourrir leurs ambitions sociales et professionnelles, d'en faire des hommes respectables et respectés.

Comme l'a souligné Claude Galarneau, les collèges classiques du milieu du $19^{\mathrm{e}}$ siècle proposent à leurs élèves différentes activités. La balle au mur figure parmi les jeux les plus anciens et demeure le plus répandu d'entre tous ${ }^{16}$. Au cours des promenades et des excursions qu'ils effectuent plus ou moins fréquemment, les élèves nagent, pêchent et font des courses. L'hiver, ils pratiquent la glissade, le patin et la raquette. Les annuaires, les témoignages d'anciens élèves ainsi que les archives institutionnelles des quatre collèges classiques pour garçons que nous avons dépouillées - le collège de Sainte-Anne de La Pocatière, les séminaires de Saint-Hyacinthe, de Nicolet et SaintCharles-Borromée de Sherbrooke - évoquent également diverses épreuves de force. Le besoin de se distraire de l'étude et de prendre l'air amène aussi la direction du collège de Sainte-Anne à mettre à contribution les étudiants pour divers travaux manuels. Pendant leurs journées de congé, ils doivent casser et transporter le tuf, une roche très friable qui se retrouve en abondance sur le terrain du collège. Cette règle obligeant les élèves à participer à l'aménagement et à l'entretien des installations extérieures existe sans doute dans d'autres institutions.

Ces activités s'inscrivent dans un idéal pédagogique destiné à former l'honnête homme, un homme complet cherchant, par sa culture et ses manières, à s'élever au-dessus de la masse populaire. Héritier de la longue tradition humaniste, le collège classique conçoit l'individu comme "un ensemble harmonieux de facultés ${ }^{17}$. Dans cette perspective, les exercices, qu'ils prennent la forme de menus travaux ou de jeux, permettent de développer les attributs physiques et d'assurer un équilibre entre l'âme et le corps. Les activités sont nécessaires pour le corps comme pour l'esprit, explique en substance

16 Galarneau, Les collèges classiques..., 201-203. Voir aussi les souvenirs de collégiens analysés par Claude Corbo dans La mémoire du cours classique. Les années aigres-douces des récits autobiographiques (Outremont : les Éditions Logiques, 2000). 17 Galarneau, Les collèges classiques..., 234. 
un règlement du collège de Sainte-Anne daté de $1845^{18}$. « La direction du Collège attache une importance toute spéciale aux amusements et jeux des élèves, tant pour favoriser le développement de leurs facultés physiques que pour sauvegarder la morale et assurer l'ardeur et l'activité dans les études », précise par ailleurs la publicité du collège Bourget de Rigaud en $1899^{19}$. Tous les élèves doivent participer avec entrain aux activités sportives rappelle, quant à lui, en 1894, un professeur du collège de Sainte-Anne ${ }^{20}$.

Dans les collèges, les jeux revêtent une autre fonction, plus informelle et implicite celle-là : ils servent d'exutoire à une certaine violence découlant des hiérarchies et des rivalités qui reproduisent plus ou moins étroitement les clivages sociaux ${ }^{21}$. La concurrence entre les élèves prend différentes formes. Elle s'exprime dans les salles de classe comme dans les cours de récréation, induit des rites d'initiation, prescrit des temps d'épreuve pour les nouveaux, contribue à établir et à maintenir des privilèges fondés sur l’âge, le degré scolaire et le statut de pensionnaire ou d'externe. Les mauvais tours, les empoignades et les bagarres à mains nues ou, même, au couteau traduisent les inimitiés et concrétisent la domination des uns sur les autres. Voici par exemple, tel que le raconte un ancien, comment se déroule la coutume de la bascule à Saint-Hyacinthe, au tournant du $20^{\mathrm{e}}$ siècle : " Quatre élèves saisissent le basculandé, l'étendent sur le dos par terre, puis le soulèvent par ses quatre membres, et après d'être approché d'une colonne ou d'un mur, ils le font balancé de toutes la force que sa résistance a mérité. À chaque coup, la base de la charpente vient heurter contre le mur ou la colonne; le supplice dure parfois plus d'une minute ${ }^{22}$. D'autres jeux, comme les batailles de balles de neige pendant la saison hivernale, expriment pareillement les rivalités. Cette violence ludique est tolérée par les directions de collège. En autant qu'elles ne provoquent pas de blessures et qu'elles ne perturbent

18 Archives de la Côte-du-Sud et du Collège Sainte-Anne (ACSA), F100 Supériorat François Pilote / 16, Avis aux écoliers sur les récréations, janvier 1845.

19 Collège Bourget, Rigaud, P.Q. : 1889-90, [s.l., s.n., 1891?], 7-8.

20 ACSA, F100/288/1 Sports, Cahier manuscrit - Compétitions sportives. Vers la même époque, on reproche à Groulx, élève au petit séminaire de Sainte-Thérèse, de ne pas jouer. Dans Lionel Groulx, Journal 1895-1911, édition critique par Giselle Huot et Réjean Bergeron, sous la direction de Benoît Lacroix, Serge Lusignan et Jean-Pierre Wallot (Montréal : Les Presses de l’Université de Montréal, 1984), tome 1, 154.

21 Voir Christine Hudon et Louise Bienvenue, « Entre franche camaraderie et amours socratiques. L'espace trouble et ténu des amitiés masculines dans les collèges classiques (1870-1960) ", Revue d'histoire de l'Amérique française, 57, 4 (printemps 2004) : 481507. L. Bienvenue et C. Hudon, «'Pour être homme, tu transgresseras'. Quelques enjeux de la socialisation masculine dans les collèges classiques québécois (1880-1939) » (à paraître dans Canadian Historical Review).

22 Archives du séminaire de Saint-Hyacinthe (ASSH), AFG 7, Mémoires de JérômeAdolphe Chicoyne (3) (d.101.6) p.D10. Nous n’avons pas corrigé la grammaire dans les passages cités. 
l'ordre des établissements, les autorités ferment en effet les yeux sur plusieurs de ces pratiques, comme les annales en font foi.

\section{L'entrée des sports modernes dans les collèges}

Après 1870, les sports, sous leur forme moderne de jeux compétitifs rigoureusement codifiés, font graduellement leur entrée dans les collèges. Divers sports collectifs, d'abord la crosse, le baseball et le hockey, puis, au tournant du $20^{\mathrm{e}}$ siècle, le football, le rugby et le basket-ball, connaissent une popularité croissante. À Saint-Hyacinthe, une première ligue de baseball est formée en 1874. Le journal Le Collégien fait ce récit imagé d'une partie particulièrement dure jouée pendant la première saison :

Un des joueurs a laissé le champ de bataille avec le doigt cassé, la mâchoire fracassée et six dents brisées. Un autre a eu la tête fendue, et quoi qu'il ne soit pas sorti de cervelle, on craint que les suites de cet accident ne soient fatales. Dans l'après-midi, les Turcos prirent une éclatante revanche, bien que le " pitcher » des adversaires employât tour à tour tous les twists magiques connus et inconnus ${ }^{23}$.

D’autres témoignages révèlent l'indéniable intérêt que suscitent les nouveaux sports. Au séminaire de Sainte-Thérèse que fréquente Lionel Groulx entre 1891-1899, " une fête spéciale des jeux », est organisée chaque année. Le programme type prévoit une des courses à obstacle, des concours athlétiques, une parade des cadets, de la musique et des chansons. Selon l'analyste de la maison, une telle manifestation est fort à propos : " elle honore et manifeste le talent des joueurs; elle consacre une exigence impérieuse dans la vie de tout homme d'étude ; elle stimule l'ardeur et le bon vouloir des élèves à s'adonner aux exercices corporels, dont la nécessité sera toujours sans conteste $»^{24}$.

Les archives des institutions collégiales recèlent par ailleurs nombre de photos de jeunes sportifs posant fièrement dans leurs uniformes, tantôt seuls, tantôt en groupe ${ }^{25}$. Des clichés montrent les installations de jeu ou, même, des joueurs en action et permettent ainsi de goûter la ferveur de certains échanges. Les cahiers et les registres des comités des sports formés dans les collèges attestent eux aussi des passions que déclenchent parfois les jeux. Le passage suivant, tiré des

23 Document cité dans ASSH, ASE012, Enseignement et vie étudiante, d.39.1, Travail de kinanthropologie de Stéfan Marchand, p. 15.

24 Cité dans Lionel Groulx, Journal 1895-1911, op. cit., 311-312.

25 Voir par exemple un échantillon de la collection du collège de Sainte-Anne disponible sur Internet, sur le site de la Société d'histoire de la Côte-du-Sud : www.Shcds.org 
archives du collège de Nicolet, met en exergue l'esprit compétitif qui anime les collégiens :

La température assez clémente, invita les Finissants et les Philos à croiser le fer pour la première fois de saison 1933-34. Les spectateurs furent tous charmés de voir que ces deux clubs se livraient une lutte à mort. Les gars de Physique gagnèrent par les points mais les Philos l'emportèrent par les poings !!

Robert Houle et Lahaie ne se ménagèrent pas les coups et les pointes plus ou moins piquantes. ${ }^{26}$

Dans le même registre, le jeune Groulx témoigne de l'attrait qu'exercent les sports chez la jeunesse étudiante, mais aussi dans la population villageoise :

Il y eut hier un " match » de Base-Ball entre les « Bingos » club formé exclusivement par les philosophes Ie année et les "Confédérés » comprenant trois représentants de chacune des classes de Rhétorique, de Belles-Lettres et de Versification. La partie fut vivement contestée et très mouvementée. Les joueurs étaient dans les meilleures conditions et une certaine animosité qui règne entre les deux clubs a contribué pour beaucoup à rendre la partie des plus intéressantes. Un grand nombre de spectateurs sont accourus du village. La victoire après avoir été vaillamment disputé est restée définitivement aux "Confédérés » ${ }^{27}$.

L'introduction des sports modernes dans les collèges résulte tantôt de l'initiative de professeurs - qui ont parfois leurs propres installations sportives ${ }^{28}$-, tantôt de revendications étudiantes. Ce sont, par exemple, des élèves d'origine américaine qui fondent la première ligue de baseball maskoutaine. Ils ont l'appui des autorités du séminaire qui fournissent aux joueurs le terrain et l'équipement. La direction des institutions acquiesce à ces nouveautés pour des raisons diverses, notamment par souci de conserver leur clientèle ou d'en attirer de nouvelles. À La Pocatière, les promenades et les courses à bicyclettes sont permises sous la pression des élèves. Dans leur requête aux directeurs, les collégiens avaient fait valoir « l'attrait » qu'exerce

26 Physique l'emporta 3 à 1 sur Philosophie. Archives du Séminaire de Nicolet (ASN), F085, série B1,1-24, activités étudiantes (comité des jeux), Livre faisant le rapport de match de hockey entre les étudiants du Séminaire, écrit par Walter Plante, élève de rhétorique et secrétaire du conseil inférieur des jeux, 26 novembre 1933, 87.

27 L. Groulx, Journal 1895-1911, p. 208-209.

28 Par exemple, à Sainte-Anne de La Pocatière, des terrains de tennis sont réservés aux prêtres. 
cette activité sur « plusieurs jeunes gens » et leur préférence pour la fréquentation $\mathrm{d}^{\prime}$ ' « un collège où on peut permettre cet exercice » ${ }^{29}$.

Au-delà, toutefois, de ces considérations d'ordre économique, l'entrée du sport dans les collèges découle d'une série de facteurs, les uns exogènes à la pastorale, les autres endogènes à celle-ci. Deux d'entre eux apparaissent particulièrement déterminants : la mutation du rapport au corps et la transformation de l'idéal viril qui l'accompagne, ainsi que le renouvellement de la spiritualité catholique. Dans cette perspective, la pratique du sport dans les collèges témoigne de la perméabilité, si relative soit-elle, de cette institution aux influences externes.

\section{Le corps sculpte l’âme}

$\mathrm{Au}$ cours des $19^{\mathrm{e}}$ et $20^{\mathrm{e}}$ siècles, les sociétés occidentales connaissent une mutation importante du rapport au corps. Comme l'ont montré, notamment, Anthony Rotundo et Donald J. Mrozek, ces transformations mènent au développement d'un ascétisme moderne associant l'équilibre et la santé à la pratique d'exercices physiques. Elles infléchissent tout particulièrement le discours sur la virilité. Selon Rotundo, un modèle de représentations valorisant l'engagement communautaire et la vie religieuse cède graduellement le pas, au cours du $19^{\text {e }}$ siècle, à une foi croissante en la réussite et en l'accomplissement personnels grâce aux talents individuels, au travail et, tout particulièrement, au contrôle de la volontéso ${ }^{\text {. Cette }}$ transformation, ayant pour toile de fond le développement de l'économie de marché, l'urbanisation et l'industrialisation, s'accompagne d'une attention croissante dévolue au corps et à l'hygiène - attention perceptible à travers les campagnes de tempérance et les appels renouvelés à la domination des pulsions sexuelles - et coïncide, par ailleurs, avec l'apparition de nouveaux héros populaires, tel Davy Crockett, modèle de robustesse, de force physique et d'endurance. L’idéal viril en émergence repose par conséquent sur un subtil mélange combinant, d'une part, puissance et vigueur musculaires et, d'autre part, maîtrise de soi. Dans ce contexte, les valeurs et les attributs militaires ont pu servir de modèle et d'inspiration aux hommes, exhortés à maintenir un équilibre entre les deux principales composantes de la nouvelle norme faisant du corps masculin à la fois une source de fierté et un sujet d'inquiétude.

Comme l'a par ailleurs démontré Donald J. Mrozek, le sport, à cette époque, est progressivement dépouillé de ses attributs péjoratifs,

29 ACSA, F100/59/XXVII Supériorat Dominique Pelletier, Requête des écoliers, 1899. 30 Antony F. Rotundo, « Body and Soul: Changing Ideals of American Middle-Class Manhood, 1770-1920 », Journal of Social History 16, 4 (Summer 1983): 23-38. 
qui en faisaient une activité dégradante et futile, pour devenir une occupation saine, respectable et productive ${ }^{31}$. Dans un climat de contention sexuelle, il en vient de plus en plus à constituer un moyen de discipliner le corps et de canaliser l'énergie sexuelle masculine. Se multiplient alors les activités sportives, tels le rugby, le football, le baseball, ainsi que les organisations et les équipes sportives, les unes laïques, les autres patronnées par des Églises. Pour plusieurs auteurs, le progrès rapide de certains sports s'insère dans un contexte de crise de la masculinité, la gymnastique et, encore davantage, le baseball et les jeux plus violents comme le rugby ayant contribué à définir un nouvel idéal viril face aux bouleversements économiques et à la montée du féminisme ${ }^{32}$. Pour d'autres historiens, le développement de ces jeux doit être interprété en termes de régulation sociale puisqu'ils ont contribué à maintenir des relations sociales stables entre les adultes, notamment en milieu ouvrier. Ils ont également coïncidé avec le déclin de la criminalité. Les sports, en effet, auraient permis de canaliser la violence des masses en la dirigeant non pas contre les élites, mais contre les pairs, sous forme d'amusements rigoureusement codifiés ${ }^{33}$.

Le Québec n'est pas étranger à ces transformations qui trouvent écho jusque dans les murs des collèges classiques masculins. L'attention plus grande dévolue au corps se manifeste notamment dans l'aménagement de l'espace. Les prospectus vantent la salubrité des lieux : " Les cours du Petit Séminaire sont vastes, il y a de l'air, de l'espace, du soleil; on y joue les grands jeux de balles (Base Ball), de ballons (Foot Ball), et de paume, etc. ${ }^{34}$. Cette assomption du corps est également perceptible à travers la mise en place de nouvelles installations sanitaires, à la fin du $19^{\mathrm{e}}$ siècle. L'eau chaude, ou à tout le moins tiède, facilite les ablutions et semble perçue par les contemporains comme un progrès majeur. La nouvelle chambre de bain est entièrement terminée, note en substance le chroniqueur du collège Sainte-Anne, en janvier 1898 : « Les élèves du cours latin ont commencé à se baigner. Ils se rendent là six ensemble à 8 h35 heures

31 Donald J. Mrozek, Sport and American Mentality, 1880-1910 (Knoxville : The University of Tennessee Press, 1983).

32 Voir les contributions de David Whitson, Bruce Kidd, Todd Crosset, Michael Kimmel, Philip G. White et Anne B. Vagi dans Michael A. Messner et Donlad F. Sabo, Sport, Men, and the Gender Order. Critical Feminist Perspectives (Champagne, Illinois : Human Kinetics Books, 1990). Une perspective d'histoire du genre oriente également ces deux études majeures portant sur le sport au Canada : Bruce Kidd, The Struggle for Canadian Sport (Toronto : University of Toronto Press, 1996); Varda Burstyn, The Rites of Men. Manhood, Politics, and the Culture of Sport (Toronto : University of Toronto Press, 1999).

33 Stephan Hardy and Alan G. Ingham, « Games, Structures and Agency: Historians on the American Play Movement », Journal of Social History 17, 2 (Winter 1983): 285301; William J. Baker, « The Making of a Working-Class Football Culture in Victorian England », Journal of Social History 13, 2 (Winter 1979): 241-51.

34 Annuaire du Petit séminaire de Ste-Marie de Monnoir, 1892-93 (Montréal : Le Séminaire, 1893), 10-11. 
du soir, un maître fait la surveillance; on met l'eau tiède. Tous les élèves devant y passer une fois par mois. [...] C'est une bien bonne amélioration ${ }^{35}$. Certes, à l'époque, la fréquence des douches ou des bains reste assez réduite. Elle augmentera peu à peu dans le demi-siècle suivant, certains établissements offrant un niveau de confort et d'hygiène plus élevé que d'autres ${ }^{36}$. Vers 1950 , les règlements de la majorité des institutions imposent dorénavant une douche hebdomadaire.

D'autres indices, d'un registre différent, témoignent d'une perception renouvelée du corps. Le roman Le beau risque du jésuite François Hertel en fait partie. Le livre, publié en 1939, exalte tout à la fois la vigueur musculaire et la maîtrise de soi. Un passage du roman, qui se déroule dans un collège, décrit les prouesses du personnage principal, un élève, qui pratique le ski : " sa jeune virilité s’affirme dans l'effort dur des montées, dans le repliement qui précède le saut, dans l'harmonieuse recherche du centre de gravité qui l'accompagne $»^{37}$.

$\mathrm{Au}$ sein de la pédagogie catholique, cette attention croissante portée au corps rejoint sur certains aspects l'humanisme traditionnel qui conçoit l'individu comme " un ensemble harmonieux de facultés ${ }^{38}$. Dans cette perspective, les sports permettent de développer les attributs physiques et d'assurer un équilibre entre l'âme et le corps. C'est ce que soulignent les Chroniques du séminaire de Sherbrooke en 1875 : « il est important de fournir aux écoliers l'occasion de prendre de l'exercice. Le corps doit prendre des forces en même temps que l'esprit acquiert des connaissances, sinon la débilité du 1er amènera l'impuissance de l'autre. C'est un principe reçu de nos jours ». On lit par ailleurs dans un prospectus du Petit Séminaire de Ste-Marie de Monnoir daté de 1893 : « on est convaincu que [...] les jeux ont pour effet d'assouplir, de fortifier le corps et de favoriser la croissance [...]. Jouer est un point de règle. Les élèves savent qu'ils sont en faute quand il ne jouent pas ${ }^{39}$. Au séminaire de Sherbrooke, en 1917, le programme de la distribution des prix pour les jeux propose les maximes suivantes :

Bien jouer, c'est à la fois développer les forces physiques, préparer une étude sérieuse et combattre puissamment l'immoralité.

\footnotetext{
35 ACSA, F100 Collège de Ste-Anne, Annales 1895-1900, 30 janvier 1898.

C. Corbo, Mémoire du cours classique, 96.

François Hertel, Le beau risque (Montréal : Éditions Bernard Valiquette, 1939), 39.

38 Galarneau, Les collèges classiques..., 234.

39 Annuaire du Petit séminaire de Ste-Marie de Monnoir, 1892-93 ( Montréal : Le Séminaire, 1893), 10-11.
} 
Les jeux donnent l'esprit de combat et de lutte qui fait les hommes vaillants que nul obstacle n'arrête.

Les jeux donnent le goût de l'endurance, apprennent la nécessité du sacrifice pour la conservation de la force ${ }^{40}$.

Cette conception est également présente dans un ouvrage du dominicain français Ferdinand-Antonin Vuillermet, paru en 1925 et dont la bibliothèque du séminaire de Saint-Hyacinthe conserve plusieurs exemplaires. Dans son livre intitulé Les jeunes gens et les sports, le Père Vuillermet définit ceux-ci comme un " exercice méthodique et hygiénique du corps humain, en vue d'accroître sa force, son adresse et sa beauté, et de développer l'énergie de la volonté tout en délassant l'esprit ${ }^{41}$. De tels propos préfigurent la conception humaniste de l'éducation physique, telle que l'a formulée, dans les années 1950, l'éducateur Antoine Paplauskas-Ramunas ${ }^{42}$.

Cette valorisation accrue du corps ne se fait toutefois pas sans heurt et sans réticences. Des critiques et des réserves se font en effet entendre au cours des premières décennies du $20^{\mathrm{e}}$ siècle. À SaintHyacinthe, l'arrivée du basket-ball pose des questions de nature morale. À cette époque, les élèves devaient porter en tout temps la redingote. Tout au plus pouvaient-ils enlever leur " capot " pour pratiquer le baseball et le hockey. En 1927, on permit enfin le short et le chandail sans manche pour la pratique du basket-ball. La nouveauté créa des remous au sein du corps enseignant. Léon Pratte, un prêtre pourtant conservateur sur de nombreuses questions, explique la situation. Loin de se scandaliser de la tenue des basketteurs, il s'amuse de l'attitude de ses confrères :

Quand apparurent, en la nouvelle salle de récréation, les équipes de ballon-panier en culotte courte, leurs bras nus, certains professeurs furent scandalisés et leur imagination de directeurs spirituels effrayée, les poussa à voir du mal là où les joueurs et leurs admirateurs n'en voyaient pas. Ce jeu de sauts et de bousculades leur paraissait favoriser la promiscuité et l'énervement des sens. L'un de ces directeurs ne voyait que de mauvais touchers. Certes, sur le plan sportif, il y en avait de nombreux, mais sur le plan moral, point ${ }^{43}$.

40 ASS, P22 / 1 Chroniques du Séminaire, 1914-1917, 216.

41 F.-A Vuillermet, Les jeunes gens et les sports ( Paris : P. Lethielleux, 1925), 11.

42 A. Paplauskas-Ramunas, L'éducation physique dans l'humanisme intégral (Ottawa: Éditions de l’Université d’Ottawa, 1954). Sur l’influence de ce livre, lire D. Guay, L'histoire de l'éducation physique, 50-52.

43 Document cité dans ASSH, ASE012, Enseignement et vie étudiante, d.39.1, Travail de kinanthropologie de Stéfan Marchand, p. 20-21. 
D'autres prêtres s'inquiètent, par ailleurs, de l'importance, à leurs yeux démesurée, que la jeunesse étudiante donne aux sports. Parmi les éducateurs, il s'en trouve en effet qui craignent que les pratiques sportives ne prennent trop de place dans la vie des élèves. Ces clercs reconnaissent aux sports d'indéniables avantages, mais redoutent que les jeunes négligent leur formation intellectuelle au profit de l'entraînement physique. En 1936, le directeur du Séminaire de SaintHyacinthe rappelle par exemple aux élèves l'importance de " conserver aux divers emplois la hiérarchie des valeurs " et souligne " que si le sport a ses raisons, il n'en demeure pas moins un moyen qu'il ne faut pas prendre pour un but ${ }^{44}$. D'autres appréhendent un développement physique désordonné. Le Père Marie-Ceslas Forest, professeur à la Faculté de philosophie de l’Université de Montréal, est de ce nombre. Il explique sa position, en 1930, dans la Revue dominicaine :

Voilà le danger pour nos collèges : faire du sport dans le but de préparer des champions universitaires. Qu'on le remarque bien, je ne condamne pas le sport professionnel. Ce que j'affirme, c'est que le sport amateur ou spécialisé va à l'encontre de la véritable éducation physique, et ne doit être introduit dans nos collèges qu'avec une extrême prudence [...] L'amateur de collège qui se modèle sur le professionnel tend de bonne heure à se spécialiser [...] Son développement physique sera donc un développement anormal, exagéré sur un point, à peu près nul sur les autres. Le sport ainsi conçu peut convenir à un professionnel qui en fait sa vie; il est simplement absurde au point de vue strictement pédagogique ${ }^{45}$.

Ce n’est donc pas en raison d’un « esprit chrétien » qui s’opposerait à un " esprit sportif » que les clercs éducateurs émettent des réserves sur la pratique du sport dans les collèges. Leur attitude découle plutôt de leur idéal de formation, où est fondamentale la notion d'équilibre entre l'âme et le corps, entre les activités intellectuelles, artistiques et physiques. C'est la crainte que le sport empiète sur la formation littéraire, scientifique, morale et religieuse, plus qu'une objection à la notion de compétition, qui motive leurs réticences. Sur un tout autre plan, mais cette objection semble secondaire, des prêtres déplorent les nombreux anglicismes que suscite l'introduction de nouveaux sports au Québec. Comme en fait foi la description d'une partie de baseball citée plus haut, le vocabulaire sportif emprunte largement à l'anglais.

\footnotetext{
44 ASSH, Chroniques du Séminaire 1929-1941, 136. Cette attitude est aussi relevée par D. Guay, L'histoire de l'éducation physique, 48-50.

45 M.-C. Forest, « Culture physique », Le Semeur, vol XXVI, nº 7 (février 1930) : p.182.
} 
Ces critiques ne doivent pas faire oublier, cependant, que la dénonciation du sport dans le discours catholique n'a aucune commune mesure avec la condamnation d'autres formes de loisirs, tel le cinéma. Ainsi, dans les années 1920 et 1930, l'Euvre des tracts publie six brochures dénonçant les effets du cinéma. Aucune brochure ne réprouve, à la même époque, les pratiques sportives. Celles-ci sont même présentées de manière essentiellement positive dans l'opuscule sur l'éducation du jésuite Wilfrid et dans la brochure sur l'œuvre des terrains de jeux fondée en $1929^{46}$.

\section{Une chrétienté combattante et virile}

Le renouvellement de la spiritualité au tournant du $20^{\mathrm{e}}$ siècle constitue un second facteur ayant favorisé une ouverture progressive du clergé enseignant à l'endroit du sport. À cet égard, la pérennité de la rhétorique sur la supériorité de l’âme, présente dans nombre de sermons et d'imprimés, a voilé les inflexions du discours clérical faisant la promotion d'une spiritualité aux accents virils, non pas fondée sur le mépris du corps, mais sur une ascèse s'attachant au contraire à le fortifier. Cette " piété virile " associe la foi à un idéal de dévouement et de grandeur d'âme, de sens du devoir et de dépassement de soi. Elle requiert de maîtriser ses pulsions, de faire de constants efforts, de chercher perpétuellement à dépasser ses propres limites. On en retrouve des manifestations dès le tout début du $20^{\mathrm{e}}$ siècle, dans le discours de l'Association catholique de la jeunesse canadiennefrançaise (ACJC) fondée en 1903, comme en témoigne cet extrait d'une allocution de $\mathrm{M}^{\text {gr }}$ Alfred Langlois aux élèves du collège de Sainte-Anne :

Nous n'avons pas reçu une éducation brillante pour être des hommes médiocres, mais pour être des hommes, des virs, comme on disait chez les Romains.

Ne craignez aucun ennemi et marchez le front haut; il n'y a que les lâches qui peuvent craindre les ennemis. Une fois que vous serez au large, avec un cœur confiant dans la miséricorde et la charité de Dieu, ne craignez aucun ennemi, ils vous seront inférieurs, continuez, continuez toujours votre marche, continuez vers les altitudes célestes ${ }^{47}$.

Ce discours est ensuite repris à compter des années 1930 par les mouvements spécialisés d'action catholique.

46 Wilfrid Lebon, L'éducation (Montréal : l'Euvre des tracts, ${ }^{\circ} 194$ (août 1935).

47 Le souligné est dans le texte original. ACSA, F100\357.1\4, Discours de $M^{g r} J$. Alfred Langlois sur l'action catholique devant les élèves de Ste Anne, 1926. 
La « piété virile » que promeut l’Église catholique de l’époque prend volontiers des accents militaires, voire guerriers. Elle puise ses métaphores tantôt dans l'imaginaire chevaleresque ou dans celui du croisé, tantôt dans un univers plus contemporain, celui de la société et des us soldatesques. C'est la religion d'un catholicisme en butte à la modernité, qui s'oppose au rationalisme, au matérialisme, au socialisme et au communisme, qui condamne les Juifs, les francsmaçons et les libres-penseurs. L’Église du Syllabus de 1864 fustigeant " les erreurs modernes », l'Église de l'encyclique Pascendi de 1907 désavouant le modernisme adopte une rhétorique militante, qui fait une large place au thème du combat ${ }^{48}$. Jean-Baptiste-Olivier Archambault, professeur de philosophie au séminaire de Saint-Hyacinthe, excite par exemple la jeunesse collégienne à la loyauté et à l'honnêteté, puis l'encourage à « combattre à son rang en bon soldat du Christ pour les grandes causes du Dieu » et à « donner sa vie » pour les êtres qui lui sont chers ${ }^{49}$. Parfois, les élèves eux-mêmes reprennent dans leurs écrits les allégories militaires. Il en est ainsi de ce finissant de 1913 :

Le collège c'est un oasis frais et riant où coule une onde limpide et pure que ne troublent les fauves, mais une onde bienfaisante sur les bords de laquelle croissent les lys et où les oiseaux du ciel viennent boire.

C'est bien de s'arrêter à cet oasis, d'y faire halte pendant un certain temps mais là n'est pas le repos définitif.

Le soldat n'est pas appelé à faire toute sa vie des exercices militaires. Il est un temps de formation. Vient la bataille. [...]

Eh bien! Moi aussi, je devrai bientôt partir... Oui partir sur la mer immense, sur la mer méchante. Dieu m'y appelle. J'irai. Il me dit qu'il y a des âmes désespérées qui vont périr sous le flot. J'irai et je les sauverai du naufrage ${ }^{50}$.

De ce discours aux accents militaires et messianiques qu'endossent l'Église et les maisons d'éducation catholiques se dégage une conception très hiérarchisée de la société chrétienne. Une grande attention est portée à la formation d'une élite catholique et apostolique. Les " chefs ", que doivent former notamment les collèges classiques à travers l'ACJC, sont des " chrétiens convaincus et des patriotes éclairés ». Ils sont aussi « des soldats » dont « le pape est le général »

48 Pour un aperçu, voir Jean Hamelin et Nicole Gagnon, Le XX $X^{e}$ siècle. Tome 1 18981940, volume III d'Histoire du catholicisme québécois (Montréal, Boréal, 1984), 178-192 notamment.

49 ASSH, ASE 14, d. 7.14, J.-B. Olivier Archambault, Ptre, « Livret remis aux finissants en 1920-1921 ».

50 ACSA, F100/75/30 Supériorat Ludger Dumais, Rév. P. Saindon, Extrait de son journal d'écolier, 22 mai 1913.

51 Hamelin et Gagnon, Le XX $X^{e}$ siècle, 191. 
Deux raisons expliquent l'apparition de cette rhétorique misant sur des valeurs dites masculines. On peut y voir, d'abord, un désir de rejoindre davantage les hommes et de leur proposer un engagement religieux qui corresponde à leurs aspirations, afin de faire contrepoids à la féminisation de la pratique religieuse, perceptible outre-Atlantique comme au Québec, même si elle se manifeste, ici, avec une moins grande ampleur ${ }^{52}$. On peut y déceler, aussi, les effets du propagandisme de l'action catholique, qui pousse à l'engagement social et au militantisme, et qui s'exerce de manière privilégiée dans la sphère publique. L'action catholique aspire en effet à une régénération religieuse et nationale. Aussi n'est-ce sans doute pas un hasard si sa symbolique recourt de préférence aux stéréotypes masculins. Sans disparaître, la sensibilité, voire la sensiblerie, qui caractérise la religion du milieu du $19^{\mathrm{e}}$ siècle, cède du terrain face à une rhétorique célébrant l'intelligence, la réflexion et le jugement ${ }^{53}$.

Dans le discours catholique, la rationalité, l'ardeur, la puissance, la combativité, la volonté, le courage et la maîtrise de soi s'incarnent dans des champs d'action et des institutions réservés aux hommes, et dont l'armée est l'expression par excellence. Quelques mois à peine après l'éclatement de la Première Guerre mondiale, Oscar Hamel, président de l'Union Régionale Québécoise de l'ACJC, propose le soldat comme figure exemplaire de discipline et d'obéissance, célèbre l'amour de l'autorité et l'esprit de sacrifice et insiste sur « l'importance de mâter sa volonté et de repousser [s]es instincts " ${ }^{54}$. Cette " piété virile », qui s'épanouit au tournant du $20^{\mathrm{e}}$ siècle, s'appuie de manière privilégiée sur la figure du Christ-roi. Ses contenus contrastent avec ceux des dévotions ultramontaines du $19^{\mathrm{e}}$ siècle qui exaltaient, à travers le culte marial et le culte à l'Enfant-Jésus, les vertus de douceur, de bonté et de pureté. Le jésuite Wilfrid Lebon note en 1935 : « n’oublions pas que le corps que l'on cultive par les exercices physiques, on ne le trouvera jamais plus beau, plus sain, mieux préparé ou plus fort et plus gracieux

52 Au Québec, le dimorphisme sexuel en matière religieuse semble toucher certaines pratiques particulières, par exemple l'adhésion aux associations pieuses ou la confession. Dans certaines régions françaises, les hommes ont quant à eux abandonné toutes pratiques et affichent un anticléricalisme plus ou moins affirmé. Philippe Boutry, dir., Du roi très chrétien à la laïcité républicaine (XVIII ${ }^{e}$ XIX ${ }^{e}$ siècles), tome 3 d'Histoire de la France religieuse, sous la dir. de Jacques Le Goff et René Rémond (Paris : Seuil, 1991); Ralph Gibson, A Social History of French Catholicism (London : Routledge, 1989); Christine Hudon, « Des dames chrétiennes. La spiritualité des catholiques québécoises au XIX ${ }^{\mathrm{e}}$ siècle ", Revue d'histoire de l'Amérique française, 49, 2 (automne 1995) : 169-194.

53 Sur la persistance des stéréotypes féminins dans le discours catholique québécois, lire Jean-François Roussel, « Roman Catholic Religious Discourse About Manhood in Quebec, From 1900 to the Quiet Revolution (1960-1980) », The Journal of Men's Studies, 11, 2 (Winter 2003) : 145.

54 ASSH, d. 8.5 dossier sur l'A.C.J.C. et sur le cercle Girouard, Oscar Hamel, « La discipline ». 
tout à la fois que dans la personne du Sauveur $»^{55}$. Le journal étudiant Le Copain, du séminaire de Sherbrooke, fait écho à cette conception christocentrique en 1945 : « Toi aussi, toi surtout, étudiant, tu as un but à atteindre : tu dois devenir un homme, un homme véritable, un homme au sens plein, au sens fort du mot, un homme façonné à l'image de celui qui a été l'Homme par excellence ».

Dans les années 1920, quelques textes font d'ailleurs la promotion du sport comme moyen d'affermir le caractère et comme outil de perfectionnement spirituel. En 1924, les périodiques français Études et la Revue des Jeunes, qui circulent au Québec, célèbrent l'ascèse de l'entraînement physique en évoquant les nombreuses analogies sportives de l'œuvre de saint Paul. Ce n'est pas l'activité physique que ces revues condamnent, mais le spectacle sportif quand il suscite l'idolâtrie et la brutalité56. Les études de Georges Hébert, un militaire français, concepteur d'une méthode d'éducation physique, font écho à une telle idée. Hébert, en effet, s’alarmait des dangers moraux des pratiques sportives. Il distinguait le sport, « dans sa conception vraie » ou éducative, et le sport " dévié ou dévoyé " ${ }^{57}$. Selon Jacques Defrance, les mêmes critiques et les mêmes réflexions traversent d'ailleurs la société allemande dans les années 1920 et $1930^{58}$.

Les propos d'Études et de la Revue des Jeunes inspirent le dominicain montréalais Marie-Ceslas Forest qui écrit en 1930 : «Par la sobriété qu'ils exigent en tout, par les saines fatigues qu'ils imposent, par l'austère discipline qu'ils inculquent ", le sport et la culture physique semblent " travailler dans le même sens que l'Église ${ }^{59}$. Comme un leitmotiv, les sermons, les allocutions et toute une littérature exhortent parents et éducateurs à développer chez les élèves certaines qualités bien précises. Lionel Groulx intitule par exemple un de ses opuscules, publié dans les années 1930, Faites-nous des Hommes. Dans ce livre, comme dans bien d'autres écrits de l'époque ${ }^{60}$, la virilité a des consonances morales et religieuses. La vigueur et la santé du corps masculin sont perçues comme le reflet de l'âme. Le célèbre adage " Mens sana in corpore sano " se décline de multiples façons. Dans un modèle de représentations qui recoupe à bien des égards les observations d'Anthony Rotundo sur l'idéal en émergence au sein de la classe moyenne américaine, l'exemple de l'homme parfait décrit par Groulx allie un esprit sain et cultivé à un corps souple, solide, discipliné et musclé. Un corps droit et ferme révèle un esprit

55 Wilfrid Lebon, L'éducation, 194.

56 ASS, P1, Le Copain, 11 novembre 1945, 1.

57 Hébert, Le Sport..., 27-67.

58 Defrance, Sociologie du sport, 21.

59 M.-C. Forest, « Culture physique », Le Semeur, vol XXVI, nº 7 (février 1930), 27. 60 Par exemple les ouvrages du dominicain Ferdinand-Antonin Vuillermet que conservent les bibliothèques de plusieurs collèges : Soyez des hommes. À la conquête de la virilité (Paris : Lethielleux, 1909); La conquête des hommes (Paris : Lethielleux, 1922). 
franc, déterminé et prêt à servir l’Église et la patrie. L'atonie musculaire témoigne au contraire d'une faiblesse de caractère. Groulx recommande ainsi :

Ayez soin de votre corps, de votre santé [...] Soignez votre maintien, votre tenue, Vous y trouverez profit physique et profit moral. A votre âge un maintien défectueux peut entraîner d'irrémédiables déformations du squelette osseux, ou d'autres troubles physiques non moins graves. L’âme sculpte le corps, chacun le sait; il faut aussi savoir que le corps sculpte l'âme. Le maintien a la vertu d'une autosuggestion. Il faut penser comme l'on veut agir. Il faut aussi se tenir comme l'on veut penser et $\operatorname{agir}^{61}$.

Le dominicain Ferdinand-Antonin Vuillermet souligne également les bienfaits moraux du sport en montrant que l'ascèse et la pratique du sport ne sont pas inconciliables, même si, à première vue, les mortifications visent à " exténuer le corps ", tandis que la culture physique cherche à l'améliorer et à " l'armer d'une puissante musculature ». L'opposition entre les deux n'est qu'apparente selon Vuillermet puisque tout sport véritable exige " une certaine mortification, et parfois bien rude, soit dans sa période d'entraînement, soit dans son exercice même $»^{62}$. Le sport comporte en fait des atouts appréciables du point de vue moral :

L'exercice des muscles a des relations très intimes avec la volonté. C'est par des actes musculaires que la volonté commence à s'essayer timidement chez l'enfant. Le long apprentissage nécessaire à chacun de nous pour que nous devenions maîtres de nos mouvements trempe notre volonté et discipline notre attention.

[...] Un effort implique le vouloir et le vouloir se développe, comme toutes nos facultés, par la répétition des actes. De plus, le travail musculaire dès qu'il tend vers la fatigue devient une douleur. Et il faut à l'homme naturellement ennemi de la peine et de la souffrance, de la volonté pour résister à la douleur et continuer, malgré les appels pressants de la paresse, l'effort commencé.

[...] L'effort, même physique, est, a-t-on dit, quelque chose d'essentiellement cérébral. Il force l'homme à vouloir, à vouloir longtemps, à se vaincre, à se posséder ${ }^{63}$.

61 Lionel Groulx, Faites-nous des Hommes. Préparation des jeunes à leurs tâches prochaines (Montréal: Les Éditions de la J.I.C., 1938), 7.

62 F.-A Vuillermet, Les jeunes gens et les sports, 25.

63 F.-A Vuillermet, Les jeunes gens et les sports, 37-38. 


\section{Conclusion}

Cet article s'est donné pour objectif de revoir l'attitude du clergé enseignant à l'égard du sport en analysant la question non pas dans une perspective binaire d'opposition/acceptation, mais en la replaçant plutôt dans un contexte plus large, celui de l'histoire des rapports au corps, et en examinant plus finement la pédagogie cléricale et le discours religieux. Notre recherche a montré que la valorisation du corps et l'introduction des sports dans les collèges se produisent bien avant 1930 et qu'elles découlent d'une conception utilitariste des exercices corporels, sensés contribuer à la maîtrise des pulsions et à la formation du caractère. Ainsi, la position du clergé enseignant à l'égard du sport et, plus largement, l'histoire du sport au Québec doivent être replacées dans la perspective d'une redéfinition de l'idéal viril à la faveur d'une transformation de l'idéal viril et du renouvellement de la spiritualité. Nous avons vu que des éducateurs émettent des réticences au sujet de la pratique des activités sportives. Ces réserves, toutefois, ne découlent guère d'un " esprit chrétien " prônant le renoncement, la mortification et le culte de l'âme, qui s'opposerait à un " esprit sportif " fondé sur la compétition. Les réticences de certains clercs résultent plutôt de leur idéal de formation, où la notion d'équilibre entre l'âme et le corps se révèle essentielle. C'est notamment la crainte que le sport empiète sur l'enseignement, plus qu'une objection à l'idée de la compétition inhérente à la pratique sportive, qui motive leurs critiques.

L'introduction de plusieurs activités sportives dans les collèges et leur articulation à l'idéal de formation sont bien sûr graduelles : on en trouve les premières traces dans la décennie 1870, mais le mouvement semble prendre de l'ampleur au début du $20^{\mathrm{e}}$ siècle. De ce point de vue, l'histoire du sport dans les institutions collégiales emprunte une chronologie semblable à celle dessinée par de nombreux auteurs analysant les sociétés américaine et canadienne ${ }^{64}$. Dans les collèges québécois comme ailleurs, l'engouement pour le hockey, le baseball, le basket-ball, notamment, donne lieu à la constitution d'équipes, à la construction d'équipements sportifs, qu'il faudrait d'ailleurs mieux documenter. Les rivalités s'expriment avec une vigueur certaine, sous le regard amusé de la jeunesse collégienne et du public villageois, sous les yeux, aussi, du clergé enseignant encourageant lui-même ces « affrontements virils ».

Ces premières décennies de l'histoire des sports modernes dans les maisons d'enseignement ont pavé la voie aux déclarations

64 Voir entre autres D. Mrozek, Sport and American Mentality...; A. Metcalfe, Canada Learns to Play; B. Kidd, The Struggle for Canadian Sport...; D. Cavallo, Muscles and Morals : organized playgrounds and urban reform 1880-1920, Philadelphia, Pennsylvania Press, 1981. 
enthousiastes publiées par des clercs au cours des années 1940 et 1950. L'extrait suivant d'un article paru dans la revue Collège et Famille donne le ton de ce discours insistant davantage encore sur les bienfaits du sport :

C'est encore par souci de la formation du caractère que le collège cherche à favoriser les jeux d'équipe, en particulier la crosse et le hockey. Jeux dynamiques qui donnent l'impression d'une bataille, mettent en exercice la passion du jeu et fournissent du coup l'occasion de se maîtriser. On y apprend à avoir l'esprit sportif, à encaisser un coup rude sans se fâcher, sans le rendre aussitôt, on vise à la beauté du jeu d'ensemble. Se montrer poli pour l'adversaire, gagner sans arrogance, subir une défaite avec bonne humeur, accepter sans la discuter la décision d'un arbitre novice, une punition imméritée, ne pas perdre la tête, se posséder enfin!....Nous ne visons pas, dans les sports, à former des professionnels du jeu, mais des hommes ${ }^{65}$.

« Former des hommes », tel était en définitive l’objectif attribué de plus en plus aux sports par les prêtres éducateurs du début du $20^{\mathrm{e}}$ siècle. Désireux de développer chez leurs élèves les qualités " viriles " - un idéal qu'épousaient au même moment de larges pans de la société - ils associaient alors étroitement « le muscle et le vouloir ».

65 Léon Massé, S.J., "Une école du caractère », Collège et famille, mai 45, 111. Citons encore cet extrait d'un article d'un journal étudiant : " Le hockey, comme tout sport d'ailleurs, concourt au développement intégral de son homme [...] Le sport, école de virilité, de discipline... ». " Yvon Bilodeau, « Par la fenêtre de sa raison », Le Copain, février 1951, 10. 